\title{
BIBECHANA
}

A Multidisciplinary Journal of Science, Technology and Mathematics

ISSN 2091-0762 (online)

Journal homepage: http://nepjol.info/index.php/BIBECHANA

\section{Structure, MESP and HOMO-LUMO study of 10-Acetyl- 10H-phenothiazine 5-oxide using vibrational spectroscopy and quantum chemical methods}

\author{
Bhawani Datt Joshi ${ }^{12^{*}}$, Poonam Tandon ${ }^{1}$, Sudha Jain ${ }^{3}$ \\ ${ }^{1}$ Department of Physics, University of Lucknow, Lucknow-226007, UP, India \\ ${ }^{2}$ Department of Physics, Siddhanath Sc. Campus, Mahendranagar, Tribhuvan University, Nepal \\ ${ }^{3}$ Department of Chemistry, University of Lucknow, Lucknow-226007, UP, India \\ "Corresponding author: E-mail: bdjoshi_007@yahoo.com \\ Article history: Received 5 October, 2012; Accepted 7 November, 2012
}

\begin{abstract}
In this communication, we have presented the geometry optimization, complete vibrational study with potential energy distribution (PED) and frontier orbital energy gap for the 10-Acetyl-10H-phenothiazine 5-oxide (APTZ) molecule using ab initio Hartree-Fock (HF) and density functional theory (DFT/B3LYP) method employing 6-311++G(d,p) basis set. The calculated IR and Raman spectra with their intensities, molecular electrostatic potential (MESP) surface and highest occupied molecular orbital (HOMO) lowest unoccupied molecular orbital (LUMO) plot have been given.
\end{abstract}

Keywords: APTZ; ab initio; DFT; IR; Raman; MESP; HOMO - LUMO.

\section{Introduction}

Phenothiazines, the heterocyclic organic compounds in which sulphur and nitrogen are incorporated in the tricyclic system, exhibit a wide range of pharmacological / biological activities [1-10]. Their several derivatives have been found to possess clinical activities, such as: tranquilizers, antihistamines, diuretics, analgesics, neurolepitcs, anticancer activity [5,6] in vitro (cancer cell lines), antileukemic [5] antimutagenic [2], anti- trypanosomal, antileishmanial [3], and inhibition of the growth of autoimmune deficiency syndrome (AIDS)-related lymphoma cells [4]. Properties of 10-H-phenothiazine, such as: semiconductivity [11], electrical conductivities [12] and low thermal activation energies of charge transfer complexes [13], has opened new practical fields of investigation. Alconea Palafox et al. [14] had given a complete vibrational analysis of the Fourier transform (FT) infrared (IR) and FT-Raman spectra of both phenothiazine (PTZ) and N-methylphenothiazine molecules using ab initio method as well as quantum chemical calculations. Sharma et al. [15] found the better biological activity of 4-thiazolidinone derivatives of phenothiazine. Dixit et al. [2] had synthesized some 10H-phenothiazine sulphone derivatives and characterized them with selected bands of IR and 1H NMR spectroscopic methods. 


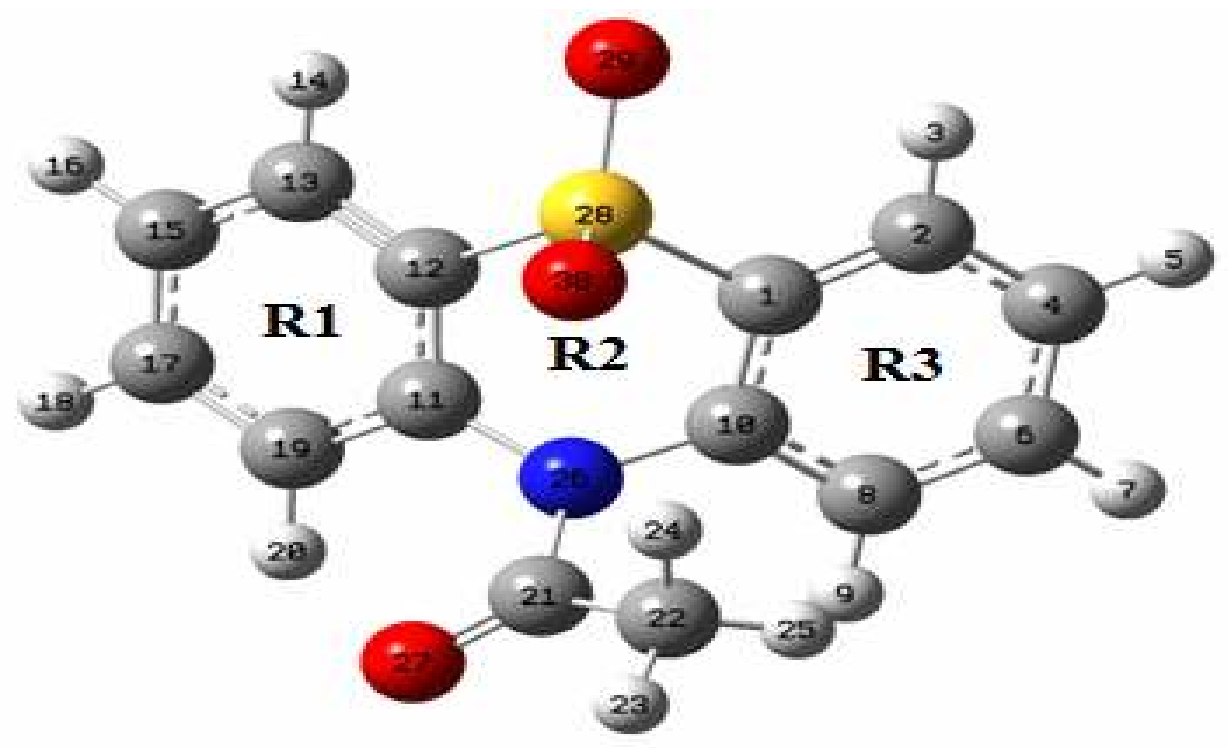

Fig. 1: Optimized structure of APTZ molecule.

Literature survey reveals that neither the vibrational assignments nor the highest molecular orbital (HOMO) - lowest unoccupied molecular orbital (LUMO) study of APTZ derivative of phenothiazine has been studied so far. Thus, a complete vibrational study along with molecular electrostatic potential (MESP) surface and HOMO - LUMO analysis has been carried in the present study using ab initio Hartree-Fock (HF) and density functional theory (DFT) method. The vibrational spectroscopy, that deals the short range structure studies, has very keen applications in these days for the structure characterization of biologically active materials [16]. HOMO - LUMO analysis has been performed which helps elucidate charge transfer occurring in the molecule.

\section{Methodology Computational}

Geometry optimization, an important issue in molecular mechanics, was performed as the first task of the computational work for the APTZ molecule taking the parameters from the X-ray diffraction data [17]. The optimized ground state molecular structure is shown in Fig. 1. The molecular structure, vibrational frequencies and energy of the optimized geometry of APTZ were computed employing the DFT [18] and HF methods using Gaussian 09 [19] program package employing 6-311++G(d,p) basis set based on Becke's three parameters (local, non-local and Hartree-Fock) hybrid exchange functional with Lee-YangParr correlation functional (B3LYP) [20,21]. The basis set $6-311++\mathrm{G}(\mathrm{d}, \mathrm{p})$ augmented by 'd' polarization functions on heavy atoms and 'p' polarization functions on hydrogen atoms as well as diffuse functions for both hydrogen and heavy atoms were used [22,23]. The absolute Raman intensities and IR absorption intensities were calculated in the harmonic approximation at the same level of theory as used for the optimized geometries associated with each normal mode, respectively. The normal mode analysis was performed and the PED was calculated along the internal coordinates using localized symmetry. For this purpose, a complete set of 84 internal coordinates were defined using Pulay's recommendations [24,25]. The vibrational assignments of the normal modes were made on the basis of the PED calculated by using the program GAR2PED [26]. Raman and IR spectra were simulated using a pure Lorentzian band profile ( $\mathrm{fwhm}=8 \mathrm{~cm}^{-1}$ ) using indigenously developed software. Visualization and confirmation of calculated data were done by using the CHEMCRAFT program [27]. 


\section{Results and Discussion}

\subsection{Geometry optimization}

Initial geometry taken from X-ray diffraction data [17] of APTZ was minimized without any constraint to the potential energy surface and the optimized structural parameters were used in the vibrational frequency calculation to characterize all stationary points as minima. The molecular conformation from the crystalline structure, as well as yielded by geometry optimization, exhibits no special symmetries. Hence, APTZ molecule crystallizes in the monoclinic, $\mathrm{P} 21 / \mathrm{n}$ form having lattice parameters a $=8.1244$ (1) $\AA, b=14.1787$ (2) $\AA, c=10.7576$ (1) $\AA, \beta=100.963(1)^{\circ}$ and $z=4$ in a unit cell [17]. The sulphoxide

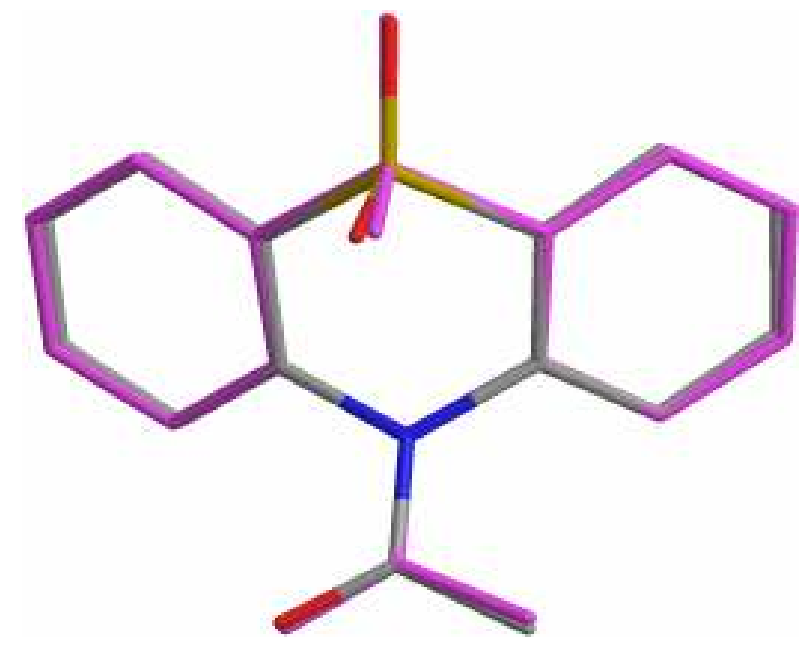

Fig. 2: Comparison of the experimental (from single crystal X-ray diffraction) and optimized structure (purple) of APTZ (hydrogen atoms are excluded for clarity).

oxygen atom is disordered over two sites with occupancies of 0.886 (4) and 0.114 (4), reflecting a partial inversion of the lone pair at the tetrahedral S-atom site. The optimized structure produced is very similar to the experimental one. Both the optimized and experimental structures of the title molecule were compared by superimposing them using a least-squares algorithm that minimizes the distances between the corresponding non-hydrogen atoms as shown in Fig. 2.

\subsection{Molecular electrostatic potential surface}

In this study, the electrostatic potential (ESP), electron density (ED) and molecular electrostatic potential (MESP) maps for APTZ are as shown in Fig. 3. In ESP, the negative potential is localized near the oxygen atoms and reflects by the yellowish blobs, while the positive potential is localized on the rest surface. However, the ED plot of the title molecule shows uniform distribution. The molecular electrostatic potential (MESP), the force acting on a positive test charge located at point through the electrical charge cloud generated through the net charge of molecule (electrons and nuclei), has been a widely used entity in the chemical literature, generally employed as a tool for probing electron rich regions [28-31]. The MESP at a point ' $r$ ' in a molecular framework with nuclear charges $Z_{\mathrm{A}}$ located at $\mathrm{R}_{\mathrm{A}}$ and electron density $\rho(r)$ is given by a relation:

$$
V(r)=\sum_{A=1}^{N} \frac{Z_{\mathrm{A}}}{\left|r-R_{\mathrm{A}}\right|}-\int \frac{\rho\left(r^{\prime}\right)}{\left|r-r^{\prime}\right|} \mathrm{d}^{3} r^{\prime}
$$


where $\mathrm{N}$ is the total number of nuclei in the molecule. The first term on the right hand side of the above equation represent the contribution due to nucleus and second due to electrons, respectively. When the latter contribution overrides the former one, the net MESP attains a negative value, providing information about electron-rich sites.

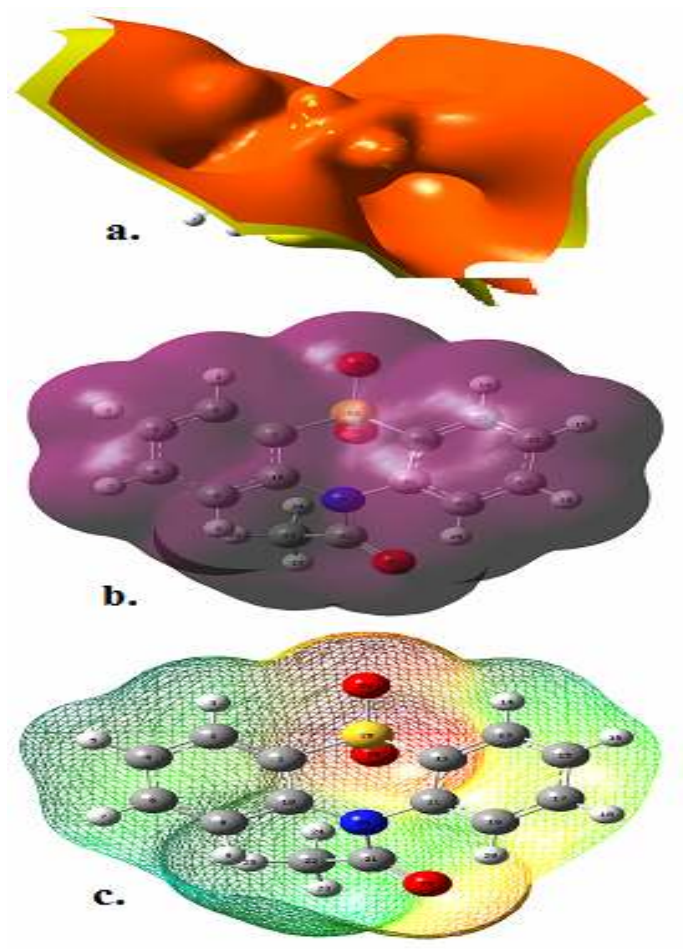

Fig. 3: (a) ESP (b) electron density (c) molecular electrostatic potential mapped on the isodensity surface in the range $\mathbf{- 6 . 5 2 1 \times 1 0 - 2}$ (red) to $\mathbf{+ 6 . 5 2 1 \times 1 0 - 2}$ (blue) for APTZ.

MESP correlates the total charge distribution with dipole moment, electronegativity, and partial charges and site of chemical reactivity of a molecule. The projection of molecular MESP of APTZ along the molecular plane is given in Fig. 3c. It provides a visual method to understand the relative polarity of a molecule and serves as a useful quantity to explain hydrogen bonding, reactivity and structure-activity relationship of molecules including biomolecules and drugs. It is the potential energy of a proton at a particular location near a molecule. Different values of the electrostatic potential at the surface of a molecule appear with the different colours. In general the attractive (or negative) potential appears in red coloured regions and those of repulsive (or positive) potential appear in blue. In the title molecule the regions near oxygen atoms are most attractive and the regions near hydrogen of methyl group are positive.

\subsection{HOMO-LUMO analysis}

The HOMO is the outermost (highest energy) orbital containing electrons that could act as an electron donor. The LUMO is the innermost (lowest energy) orbital that has room to accept electrons and can act as the electron acceptor. According to the frontier molecular orbital theory, the formation of a transition state is due to an interaction between the frontier orbitals (HOMO and LUMO) of reactants [32]. The energy of the HOMO is directly related to the ionization potential and the energy of the LUMO is directly related to the electron affinity. High value of HOMO energy is likely to indicate a tendency of the molecule to donate electrons to appropriate acceptor molecule of low empty molecular orbital energy. The lower values of LUMO energy show more probability to accept electrons. So, the gap energy, i.e. the 
difference in energy between the HOMO and LUMO, is an important stability index. It is a critical parameter in determining molecular electrical transport properties because it is a measure of electron conductivity. Low gap value refers to the higher electronic transition and vice versa. The HOMO - LUMO plot with the frontier orbital energy gap for the title molecule is shown in Fig. 4.

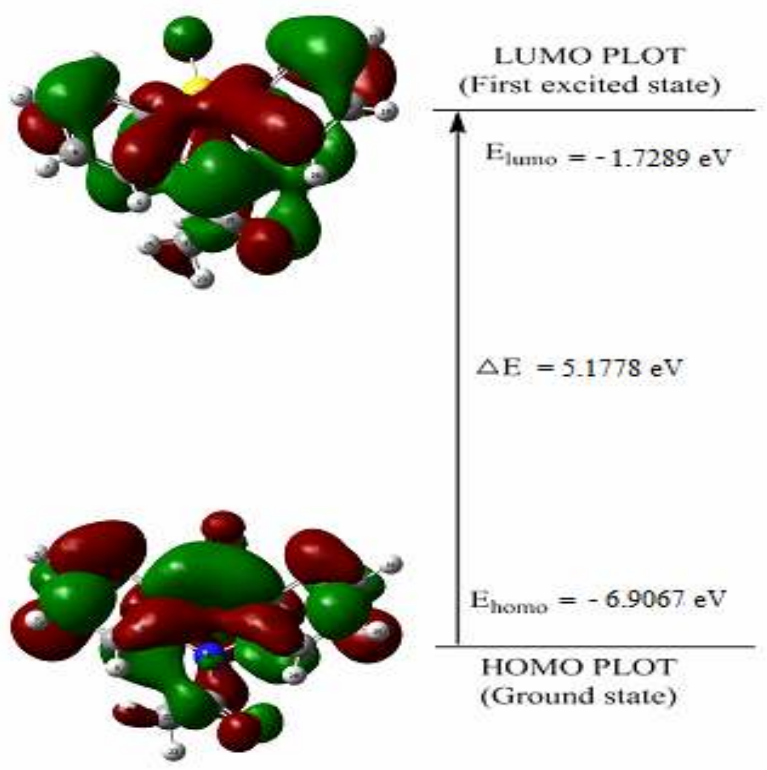

Fig. 4: HOMO-LUMO plot of APTZ molecule.

In HOMO the main electronic transition is occurred at $\mathrm{C}=\mathrm{C}$ bonds of all the rings, $\mathrm{S}$ atom of the rings $\mathrm{R} 2$ and carbonyl group (in small amounts), while in LUMO the charge density is mainly accumulated at CC bond in rings.

Table 1. Electronic transitions, absorption wavelength $\lambda_{\max }(\mathrm{nm})$, excitation energy $(\mathrm{eV})$, oscillator strengths (f), frontier orbital energies (eV) and dipole moment (Debye) of APTZ.

\begin{tabular}{|c|c|c|c|c|c|c|c|c|c|}
\hline \multirow[b]{3}{*}{$\begin{array}{c}\text { Excited } \\
\text { States }\end{array}$} & \multicolumn{8}{|c|}{ Calculated } & \multirow{3}{*}{$\begin{array}{l}\text { Transition } \\
\text { type/ } \\
\text { assignments }\end{array}$} \\
\hline & \multicolumn{4}{|c|}{ Gas Phase } & \multicolumn{4}{|c|}{ Benzene solution } & \\
\hline & $\begin{array}{l}\lambda_{\max } \\
(\mathrm{nm})\end{array}$ & Transitions & $\mathrm{E}(\mathrm{ev})$ & $\begin{array}{l}\text { Oscillator } \\
\text { strength } \\
\text { (f) }\end{array}$ & $\begin{array}{l}\lambda_{\max } \\
(\mathrm{nm})\end{array}$ & Transitions & $\mathrm{E}(\mathrm{ev})$ & $\begin{array}{l}\text { Oscillator } \\
\text { strength } \\
\text { (f) }\end{array}$ & \\
\hline 1 & 278 & $\mathrm{H} \rightarrow \mathrm{L}$ & 4.4594 & 0.0549 & 280 & $\mathrm{H} \rightarrow \mathrm{L}$ & 4.4249 & 0.0838 & $\pi \rightarrow \pi^{*}$ \\
\hline 2 & 256 & $\mathrm{H}-1 \rightarrow \mathrm{L}$ & 4.8445 & 0.0152 & 251 & $\mathrm{H} \rightarrow \mathrm{L}+1$ & 4.9486 & 0.0983 & \\
\hline 3 & 252 & $\mathrm{H} \rightarrow \mathrm{L}+1$ & 4.9107 & 0.0537 & 246 & $\mathrm{H}-3 \rightarrow \mathrm{L}$ & 5.0408 & 0.0271 & \\
\hline 4 & 244 & $\mathrm{H} \rightarrow \mathrm{L}+2$ & 5.0754 & 0.0283 & 244 & $\mathrm{H} \rightarrow \mathrm{L}+2$ & 5.0852 & 0.0489 & $\pi \rightarrow \pi^{*}$ \\
\hline 5 & 236 & $\mathrm{H}-3 \rightarrow \mathrm{L}$ & 5.2463 & 0.0081 & 235 & $\mathrm{H}-5 \rightarrow \mathrm{L}$ & 5.2858 & 0.0160 & \\
\hline 6 & 231 & $\mathrm{H}-5 \rightarrow \mathrm{L}$ & 5.3783 & 0.0091 & 225 & $\mathrm{H} \rightarrow \mathrm{L}+3$ & 5.4995 & 0.0215 & \\
\hline 7 & 226 & $\mathrm{H}-7 \rightarrow \mathrm{L}$ & 5.4730 & 0.0122 & 222 & $\mathrm{H} \rightarrow \mathrm{L}+4$ & 5.5930 & 0.0434 & \\
\hline \multirow[t]{2}{*}{8} & 221 & $\mathrm{H}-1 \rightarrow \mathrm{L}+2$ & 5.154 & 0.0152 & 221 & $\mathrm{H}-6 \rightarrow \mathrm{L}$ & 5.6169 & 0.0224 & \\
\hline & \multicolumn{2}{|c|}{$\mathrm{E}_{\text {HOMO }}(\mathrm{eV})$} & \multicolumn{2}{|c|}{$\mathrm{E}_{\text {LUMO }}(\mathrm{eV})$} & $\Delta \mathrm{E}(\mathrm{eV})$ & \multicolumn{2}{|l|}{$\mu(D)$} & & \\
\hline Gas & \multirow{2}{*}{\multicolumn{2}{|c|}{-6.9067143}} & \multicolumn{2}{|c|}{-1.7289234} & 5.1777909 & \multicolumn{2}{|l|}{6.1429} & & \\
\hline Benzene & & & -1.7066 & & 5.1573834 & \multicolumn{2}{|l|}{7.0474} & & \\
\hline
\end{tabular}


The calculated frontier orbital energies, absorption wavelengths $\left(\lambda_{\max }\right)$, oscillator strengths (f), excitation energies (E) and dipole moments $(\mu)$ for gas phase and benzene solvent environment (integral equation formalism-polarizable continuum model, IEF-PCM) using the TD-DFT/6-31G method are illustrated in Table 1. This electronic absorption corresponds to the transition from the ground to the first excited state and is mainly described by one electron excitation from the HOMO to the LUMO. The first alloweddipole transition in the gas phase was calculated about $278 \mathrm{~nm}$ with oscillator strength 0.0549 . The next transitions were calculated about 252, 244 and $221 \mathrm{~nm}$ with oscillator strengths $0.0537,0.0283$ and 0.0152 , respectively. The transitions $\pi \rightarrow \pi^{*}$ are the main observed transitions.

\subsection{Vibrational assignment}

An APTZ molecule has 30 atoms and hence gives 84 (3N-6) fundamental modes of vibration. All of them are both the Raman and IR active. Since the vibrational wavenumbers calculated by DFT methods are higher than their precise values, so they were scaled down by the wavenumber linear scaling procedure (WLS) $\left[v_{\mathrm{obs}} / v_{\mathrm{cal}}=\left(1.0087-0.0000163 \mathrm{x} \mathrm{v} \mathrm{v}_{\mathrm{cal}}\right) \mathrm{cm}^{-1}\right]$ of Yoshida et al. [33]. However, there are different scaling factors, but the vibrational wavenumbers calculated uniformly scaled with only one scaling factor $[34,35]$ are often in good agreement to the observed ones.

The Raman scattering cross sections, $\partial \sigma_{\mathrm{j}} / \partial \Omega$, which are proportional to the Raman intensities, may be calculated from the Raman scattering amplitude and predicted wavenumbers for each normal modes using the relationship [36,37]

$$
\frac{\partial \sigma_{j}}{\partial \Omega}=\left(\frac{2^{4} \pi^{4}}{45}\right)\left(\frac{\left(v_{0}-v_{j}\right)^{4}}{1-\exp \left[\frac{-h c v_{j}}{k T}\right]}\right)\left(\frac{h}{8 \pi^{2} c v_{j}}\right) S_{j}
$$

where $S_{\mathrm{j}}$ and $v_{\mathrm{j}}$ are the calculated scattering activities and the predicted wavenumbers, respectively, of the $\mathrm{j}^{\text {th }}$ normal mode, $\mathrm{v}_{\mathrm{o}}$ is the Raman excitation wavenumber and $\mathrm{h}, \mathrm{c}$ and $\mathrm{k}$ are the universal constants.

Assignments have been made on the basis of relative intensities, energies, line shape and potential energy distribution. All the vibrational bands have been assigned satisfactorily, together with the IR and the Raman intensities. The assigned wavenumbers of the vibrational modes calculated at the HF and B3LYP level with the basis set 6-311++G(d,p) along with their PED are given in Table 2 .

In the APTZ molecule, there are three rings with different functional groups as shown in Fig. 1. The vibrational assignments of these rings along with some functional groups have been discussed separately. The calculated IR and Raman spectra are given in the Figs. 5 and 6, respectively.

\subsubsection{CH3 vibrations}

The title molecule has one methyl group associated with different types of vibrations: like symmetric and asymmetric stretching, deformations and rocking as listed in Table 2. Asymmetric stretching of CH3 is at $3039 / 3017 \mathrm{~cm}^{-1}$ in scaled DFT having intensities of 5.43/3.53 a.u in the IR and 258.68/292.55 a.u in the Raman spectra, respectively. The symmetric stretching is calculated at $2927 \mathrm{~cm}-1$ (contribution 100\%). This band is weak in IR band with intensity of 2.81 a.u. and strong intensity of 824.08 a.u. in the Raman spectra. The asymmetric bending modes of this group are calculated at 1461 and $1447 \mathrm{~cm}^{-1}$, while the symmetric bending mode is calculated around $1380 \mathrm{~cm}^{-1}$. The rocking vibration is calculated at 1043 and $1017 \mathrm{~cm}^{-1}$ in the scaled DFT, which is weaker in intensity in the IR spectrum in comparison to that in the Raman spectrum. 
Bhawani Datt Joshi et al. / BIBECHANA 9 (2013) 38-49: BMHSS, p.44 (Online Publication: Nov., 2012)

Table 2. Calculated wavenumbers (in $\mathrm{cm}^{-1}$ ), IR and Raman intensities (in a.u.).

\begin{tabular}{|c|c|c|c|c|c|}
\hline Unscaled & \multicolumn{2}{|c|}{ Scaled } & \multicolumn{2}{|c|}{ Intensity } & \multirow[t]{2}{*}{ PEDa $(\%)$} \\
\hline DFT & HF & DFT & IR & Raman & \\
\hline 3232 & 3228 & 3090 & 0.31 & 382.37 & $\mathrm{R} 1[\mathrm{v}(\mathrm{CH})](97)$ \\
\hline 3203 & 3213 & 3063 & 6.21 & 1517.81 & $\mathrm{R} 3[\mathrm{v}(\mathrm{CH})](96)$ \\
\hline 3202 & 3212 & 3063 & 8.53 & 585.95 & $\mathrm{R} 1[\mathrm{v}(\mathrm{CH})](95)$ \\
\hline 3198 & 3205 & 3059 & 2.15 & 174.26 & $\mathrm{R} 3[\mathrm{v}(\mathrm{CH})](98)$ \\
\hline 3190 & 3197 & 3052 & 6.43 & 696.38 & $\mathrm{R} 1[\mathrm{v}(\mathrm{CH})](98)$ \\
\hline 3189 & 3196 & 3051 & 5.87 & 487.54 & $\mathrm{R} 3[\mathrm{v}(\mathrm{CH})](99)$ \\
\hline 3176 & 3182 & 3040 & 3.5 & 309.77 & $\mathrm{R} 1[\mathrm{v}(\mathrm{CH})](99)$ \\
\hline 3176 & 3181 & 3039 & 1.74 & 271.13 & $\mathrm{R} 3[\mathrm{v}(\mathrm{CH})](99)$ \\
\hline 3151 & 3152 & 3017 & 5.43 & 258.68 & va(CH3)(99) \\
\hline 3120 & 3123 & 2988 & 3.53 & 292.55 & $\mathrm{v}_{\mathrm{a}}\left(\mathrm{CH}_{3}\right)(100)$ \\
\hline 3052 & 3061 & 2927 & 2.81 & 824.08 & $v_{\mathrm{s}}\left(\mathrm{CH}_{3}\right)(100)$ \\
\hline 1760 & 1906 & 1725 & 321.27 & 503.6 & {$[v(\mathrm{C}=\mathrm{O})](78)+\rho[\mathrm{C}=\mathrm{O}](7)+[\mathrm{v}(\mathrm{CC})](5)$} \\
\hline 1636 & 1750 & 1607 & 18.47 & 370.17 & $\mathrm{R} 3\left[\mathrm{v}(\mathrm{CC})(32)+\delta_{\text {in }}(\mathrm{CH})(6)\right]+\mathrm{R} 1\left[\mathrm{v}(\mathrm{CC})(22)+\delta_{\mathrm{a}}(5)\right]$ \\
\hline 1624 & 1742 & 1595 & 32.61 & 1411.67 & $\mathrm{R} 1\left[\mathrm{v}(\mathrm{CC})(35)+\delta_{\text {in }}(\mathrm{CH})(11)+\delta_{\mathrm{a}}(6)\right]+\mathrm{R} 3[\mathrm{v}(\mathrm{CC})](27)$ \\
\hline 1619 & 1733 & 1591 & 13.48 & 527.92 & $\left.\mathrm{R} 1\left[\mathrm{v}(\mathrm{CC})(35)+\delta^{\prime} \mathrm{a}(5)\right]+\mathrm{R} 3[\mathrm{v}(\mathrm{CC})](27)+\mathrm{R} 2[\mathrm{v}(\mathrm{CC})] 7\right)$ \\
\hline 1609 & 1719 & 1581 & 2.36 & 178.71 & $\left.\mathrm{R} 1[\mathrm{v}(\mathrm{CC})](31)+\mathrm{R} 3\left[\mathrm{v}(\mathrm{CC})(27)+\delta_{\text {in }}(\mathrm{CH})\right](8)\right]+\mathrm{R} 2[\mathrm{v}(\mathrm{CC})](9)$ \\
\hline 1507 & 1610 & 1483 & 52.39 & 7.75 & $\begin{array}{l}\left.\left.\mathrm{R} 1\left[\delta_{\text {in }}(\mathrm{CH})(25)+\mathrm{v}(\mathrm{CC})\right](11)\right]+\mathrm{R} 3\left[\delta_{\text {in }}(\mathrm{CH})\right](24)+\mathrm{v}(\mathrm{CC})\right](6)+\mathrm{R} 2[\mathrm{v}(\mathrm{CC})] \\
(8)\end{array}$ \\
\hline 1492 & 1595 & 1468 & 90.55 & 105.5 & $\begin{array}{l}\mathrm{R} 1\left[\delta_{\text {in }}(\mathrm{CH})(25)+\mathrm{v}(\mathrm{CC})(19)\right]+\mathrm{R} 3\left[\delta_{\text {in }}(\mathrm{CH})\right](20)+\mathrm{R} 2[\mathrm{v}(\mathrm{CC})(8)+\mathrm{v}(\mathrm{CN})(8 \\
)]\end{array}$ \\
\hline 1484 & 1578 & 1461 & 18.95 & 202.89 & {$\left[\delta_{\mathrm{a}}(60)+\delta_{\mathrm{a}}{ }_{\mathrm{a}}(22)+\rho^{\prime}(6)\right]\left(\mathrm{CH}_{3}\right)$} \\
\hline 1481 & 1574 & 1458 & 13.74 & 21.16 & $\mathrm{R} 3\left[\delta_{\text {in }}(\mathrm{CH})(33)+\mathrm{v}(\mathrm{CC})(21)\right]+\mathrm{R} 1\left[\delta_{\text {in }}(\mathrm{CH})\right](14)+\mathrm{R} 2\left[\delta_{\text {trig }}\right](7)$ \\
\hline 1476 & 1572 & 1454 & 5.69 & 26.18 & $\left.\mathrm{R} 1\left[\delta_{\text {in }}(\mathrm{CH})(38)+\mathrm{v}(\mathrm{CC})(22)\right]+\mathrm{R} 3\left[\delta_{\text {in }}(\mathrm{CH})\right](11)+\mathrm{v}(\mathrm{CC})(8)\right]$ \\
\hline 1469 & 1559 & 1447 & 7.43 & 128.29 & {$\left[\delta^{\prime}(61)+\delta_{\mathrm{a}}(19)+\rho(9)\right]\left(\mathrm{CH}_{3}\right)$} \\
\hline 1399 & 1509 & 1380 & 40.71 & 79.17 & $\delta_{\mathrm{s}}\left[\mathrm{CH}_{3}\right](88)+[\mathrm{v}(\mathrm{CC})](7)$ \\
\hline 1340 & 1427 & 1323 & 12.27 & 376.6 & $\mathrm{R} 1[\mathrm{v}(\mathrm{CC})](49)+\mathrm{R} 3[\mathrm{v}(\mathrm{CC})](26)+\mathrm{R} 2[\mathrm{v}(\mathrm{CC})] 6)$ \\
\hline 1332 & 1405 & 1315 & 62.21 & 319.78 & $\mathrm{R} 2\left[\mathrm{v}(\mathrm{CN})(26)+\delta_{\text {in }}(\mathrm{NC} 21)(6)\right]+\mathrm{R} 3\left[\mathrm{v}(\mathrm{CC})(24)+\delta_{\text {in }}(\mathrm{CH})(7)\right]$ \\
\hline 1326 & 1396 & 1309 & 10.69 & 307.35 & $\mathrm{R} 1\left[\mathrm{v}(\mathrm{CC})(36)+\delta_{\text {in }}(\mathrm{CH})(8)\right]+\mathrm{R} 3\left[\mathrm{v}(\mathrm{CC})(25)+\delta_{\text {in }}(\mathrm{CH})(9)\right]+\mathrm{R} 2[\mathrm{v}(\mathrm{CC})](9)$ \\
\hline 1309 & 1369 & 1293 & 61.86 & 190.42 & $\begin{array}{l}\left.\mathrm{R} 1\left[\delta_{\text {in }}(\mathrm{CH})\right](25)+\mathrm{v}(\mathrm{NC} 21)(11)\right]+\mathrm{R} 3\left[\delta_{\text {in }}(\mathrm{CH})(22)+\mathrm{v}(\mathrm{CC})(5)\right]+\mathrm{R} 2\left[\delta_{\text {trig }}\right]( \\
13)\end{array}$ \\
\hline 1272 & 1345 & 1257 & 277.81 & 400.6 & $\mathrm{R} 2\left[\mathrm{v}_{\mathrm{a}}\left(\mathrm{SO}_{2}\right)\right](85)$ \\
\hline 1257 & 1308 & 1243 & 97.12 & 1261.13 & $\mathrm{R} 2\left[\mathrm{v}(\mathrm{NC} 21)(16)+\delta_{\text {in }}(\mathrm{CH})(18)+\mathrm{v}(\mathrm{CC})(8)+\mathrm{v}(\mathrm{CN})(22)\right]$ \\
\hline 1256 & 1300 & 1241 & 134.26 & 625.29 & $\left.\mathrm{R} 3\left[\delta_{\text {in }}(\mathrm{CH})\right](26)+\mathrm{v}(\mathrm{CC})(11)\right]+\mathrm{R} 2[\mathrm{v}(\mathrm{CN})](17)+\mathrm{R} 1[\mathrm{v}(\mathrm{NC} 21)](13)$ \\
\hline 1213 & 1294 & 1200 & 64.05 & 1000.42 & $\begin{array}{l}\mathrm{R} 2\left[\mathrm{v}(\mathrm{CN})(15)+\mathrm{v}(\mathrm{CC})(12)+\delta_{\text {in }}(\mathrm{CH})(11)+\mathrm{v}(\mathrm{NC} 21)(7)+\delta_{\text {trig }}(6)\right]+[\mathrm{v}(\mathrm{CC})] \\
(11)+\delta[\mathrm{C}=\mathrm{O}](9)+\left[\rho\left[\mathrm{CH}_{3}\right](5)\right.\end{array}$ \\
\hline 1187 & 1251 & 1174 & 0.46 & 62.57 & $\mathrm{R} 1\left[\delta_{\text {in }}(\mathrm{CH})(55)+\mathrm{v}(\mathrm{CC})(9)\right]+\mathrm{R} 3\left[\delta_{\text {in }}(\mathrm{CH})(19)+\delta_{\text {in }}(\mathrm{CH})(9)\right]$ \\
\hline 1186 & 1233 & 1173 & 0.71 & 356.79 & $\mathrm{R} 3\left[\delta_{\text {in }}(\mathrm{CH})(54)+\mathrm{v}(\mathrm{CC})(10)\right]+\mathrm{R} 1\left[\delta_{\text {in }}(\mathrm{CH})\right](23)$ \\
\hline 1159 & 1213 & 1147 & 41.18 & 654.94 & $\begin{array}{l}\mathrm{R} 1\left[\delta_{\text {in }}(\mathrm{CH})(22)+\mathrm{v}(\mathrm{CC})(20)\right]+\mathrm{R} 3\left[\delta_{\text {in }}(\mathrm{CH})(15)+\mathrm{v}(\mathrm{CC})(11)\right]+\mathrm{R} 2[\mathrm{v}(\mathrm{CS})]( \\
13)\end{array}$ \\
\hline 1153 & 1188 & 1142 & 3.5 & 123.02 & $\mathrm{R} 3\left[\delta_{\text {in }}(\mathrm{CH})(23)+\mathrm{v}(\mathrm{CC})(21)\right]+\mathrm{R} 1\left[\mathrm{v}(\mathrm{CC})(15)+\delta_{\text {in }}(\mathrm{CH})(15)\right]+\mathrm{R} 2[\mathrm{v}(\mathrm{CS})](10)$ \\
\hline 1124 & 1185 & 1113 & 123.48 & 1162.02 & $\mathrm{R} 2\left[v_{\mathrm{s}}\left(\mathrm{SO}_{2}\right)(32)+\mathrm{v}(\mathrm{CS})(12)\right]+\mathrm{R} 1\left[\delta_{\text {trig }}\right](9)+\mathrm{R} 3\left[\delta_{\text {trig }}\right](9)$ \\
\hline 1083 & 1159 & 1073 & 26.3 & 29.9 & $\begin{array}{l}\mathrm{R} 3\left[\delta_{\text {trig }}(18)+\delta_{\text {in }}(\mathrm{CH})(8)+v(\mathrm{CC})(7)\right]+\mathrm{R} 1\left[\delta_{\text {trig }}(19)+\mathrm{v}(\mathrm{CC})(6)\right]+\mathrm{R} 2[\mathrm{v}(\mathrm{CS})](1 \\
3)\end{array}$ \\
\hline 1058 & 1149 & 1049 & 37.24 & 1602.59 & $\begin{array}{l}\mathrm{R} 1[\mathrm{v}(\mathrm{CC})](22)+\delta_{\text {in }}(\mathrm{CH})(8)+\left[\rho^{\prime}(13)+\rho(8)\right](\mathrm{CH} 3)+\mathrm{R} 2\left[\mathrm{v}_{\mathrm{s}}\left(\mathrm{SO}_{2}\right)\right](14)+\omega[ \\
\mathrm{C}=\mathrm{O}](5)+\left[\mathrm{R} 3\left[\mathrm{v}(\mathrm{CC})(5)+\delta_{\text {trig }}(5)\right]\right.\end{array}$ \\
\hline 1057 & 1144 & 1048 & 0.46 & 248.47 & $\mathrm{R} 3\left[\mathrm{v}(\mathrm{CC})(37)+\delta_{\text {in }}(\mathrm{CH})(10)+\delta_{\text {trig }}(7)\right]+\mathrm{R} 1\left[\mathrm{v}(\mathrm{CC})(21)+\delta_{\text {trig }}(9)\right]$ \\
\hline
\end{tabular}


Bhawani Datt Joshi et al. / BIBECHANA 9 (2013) 38-49: BMHSS, p.45 (Online Publication: Nov., 2012)

\begin{tabular}{|c|c|c|c|c|c|}
\hline 1052 & 1115 & 1043 & 0.59 & 839.1 & {$\left[\rho^{\prime}(43)+\rho(7)+\delta_{a}(5)\right]\left(\mathrm{CH}_{3}\right)+\omega[\mathrm{C}=\mathrm{O}](12)+\mathrm{R} 1[\mathrm{v}(\mathrm{CC})](6)$} \\
\hline 1048 & 1113 & 1039 & 42.63 & 1154.47 & $\mathrm{R} 2\left[\mathrm{v}_{\mathrm{s}}\left(\mathrm{SO}_{2}\right)\right](31)+\mathrm{R} 3\left[\delta_{\text {trig }}(10)+\mathrm{v}(\mathrm{CC})(8)\right]+\mathrm{R} 1\left[\delta_{\text {trig }}(17)+\mathrm{v}(\mathrm{CC})(8)\right]$ \\
\hline 1026 & 1107 & 1017 & 32.89 & 197.49 & $\begin{array}{l}{\left[\rho(38)+\rho^{\prime}(10)\right]\left(\mathrm{CH}_{3}\right)+\mathrm{R} 1\left[\delta_{\text {trig }}(11)+v(\mathrm{NC} 21)(7)\right]+\rho[\mathrm{C}=\mathrm{O}](5)+[\mathrm{v}(\mathrm{CC})](} \\
5)+\delta{ }^{\prime} \mathrm{a}\left[\mathrm{CH}_{3}\right](5)\end{array}$ \\
\hline 1003 & 1106 & 996 & 0.05 & 6.51 & $\mathrm{R} 3[\mathrm{oop}(\mathrm{CH})(81)+\operatorname{puck}(12)]$ \\
\hline 1002 & 1103 & 994 & 0.09 & 6.74 & $\mathrm{R} 1[\mathrm{oop}(\mathrm{CH})(83)+\operatorname{puck}(11)]$ \\
\hline 991 & 1078 & 984 & 2.79 & 197.74 & $\begin{array}{l}\mathrm{R} 3\left[\delta_{\text {trig }}\right](25)+\mathrm{R} 1[\operatorname{oop}(\mathrm{CH})(12)]+\mathrm{R} 2\left[\delta_{\text {trig }}(6)+\delta_{\text {in }}(\mathrm{NC} 21)(6)\right]+[\mathrm{v}(\mathrm{CC})](8) \\
+\rho[\mathrm{C}=\mathrm{O}](6)\end{array}$ \\
\hline 974 & 1076 & 967 & 0.87 & 13.73 & $\mathrm{R} 3\left[\mathrm{oop}(\mathrm{CH})(88)+\tau^{\prime}(5)\right]$ \\
\hline 970 & 1057 & 963 & 0.85 & 43.24 & $\left.\mathrm{R} 1[\mathrm{oop}) \mathrm{CH})(84)+\tau^{\prime}(5)\right]$ \\
\hline 907 & 988 & 902 & 7.46 & 106.16 & $\begin{array}{l}{[v(\mathrm{CC})](20)+\mathrm{R} 1\left[\delta_{\text {trig }}(15)+\mathrm{v}(\mathrm{CC})\right](7)+\mathrm{R} 3[\mathrm{oop}(\mathrm{CH})](7)+\mathrm{R} 2[\mathrm{v}(\mathrm{CN})](6)+} \\
\rho\left[\mathrm{CH}_{3}\right](5)\end{array}$ \\
\hline 888 & 978 & 883 & 0.14 & 24.54 & $\mathrm{R} 3[\operatorname{oop}(\mathrm{CH})(81)+\operatorname{puck}(5)]$ \\
\hline 883 & 960 & 878 & 0.43 & 45.94 & $\mathrm{R} 1[\mathrm{oop}(\mathrm{CH})(85)+\operatorname{puck}(7)]$ \\
\hline 780 & 856 & 777 & 65.95 & 18.38 & $\mathrm{R} 3[\mathrm{oop}(\mathrm{CH})(53)+\operatorname{puck}(7)]+\mathrm{R} 1[\mathrm{oop}(\mathrm{CH})](16)+\mathrm{R} 2[\mathrm{puck}](6)$ \\
\hline 772 & 848 & 769 & 7.95 & 8.61 & $\mathrm{R} 1[\mathrm{oop}(\mathrm{CH})](61)+\mathrm{R} 3[\mathrm{oop}(\mathrm{CH})](22)$ \\
\hline 760 & 838 & 757 & 13.83 & 205.58 & $\mathrm{R} 3[\operatorname{puck}(39)+\mathrm{oop}(\mathrm{CH})(8)]+\mathrm{R} 1[$ puck] $(17)+\mathrm{R} 2[$ puck] $(17)$ \\
\hline 744 & 812 & 741 & 29.41 & 38.51 & $\left.\mathrm{R} 1[\operatorname{puck}(25)+\mathrm{oop}(\mathrm{CH})(7)]+\mathrm{R} 3\left[\delta_{\mathrm{a}}(14)+\operatorname{puck}(10)\right]+\mathrm{R} 2[\mathrm{v}(\mathrm{CS})] 7\right)$ \\
\hline 740 & 808 & 737 & 2.75 & 49.1 & $\begin{array}{l}\mathrm{R} 1\left[\delta_{\mathrm{a}}(16)+\delta_{\mathrm{a}}(5)+\operatorname{puck}(12)+\mathrm{R} 3\left[\delta_{\mathrm{a}}(11)+\delta_{\mathrm{a}}{ }(5)+\operatorname{puck}(7)\right]+\mathrm{R} 2[\mathrm{v}(\mathrm{CS})(7)\right. \\
+\mathrm{v}(\mathrm{NC} 21)(5)]\end{array}$ \\
\hline 732 & 795 & 730 & 41.02 & 52.37 & $\mathrm{R} 1\left[\operatorname{puck}(24)+\delta^{\prime}{ }_{\mathrm{a}}(6)\right]+\mathrm{R} 3[$ puck $](21)+\mathrm{R} 2\left[\omega\left(\mathrm{SO}_{2}\right)(8)+\mathrm{v}(\mathrm{CS})(10)+\tau(6)\right]$ \\
\hline 680 & 734 & 678 & 0.96 & 1560.6 & $\mathrm{R} 1\left[\delta_{\mathrm{a}}{ }_{\mathrm{a}}(24)+\delta_{\mathrm{a}}(5)\right]+\mathrm{R} 3\left[\delta^{\prime}{ }_{\mathrm{a}}(17)+\delta_{\mathrm{a}}(10)+\operatorname{puck}(5)\right]+\mathrm{R} 2[\mathrm{v}(\mathrm{CS})](8)$ \\
\hline 650 & 696 & 649 & 6.25 & 59.28 & $\mathrm{R} 3\left[\delta_{\mathrm{a}}{ }^{\prime}\right](39)+\mathrm{R} 1\left[\delta_{\mathrm{a}}(15)+\delta^{\prime}{ }_{\mathrm{a}}(8)\right]+\rho[\mathrm{C}=\mathrm{O}](7)+[\mathrm{v}(\mathrm{CC})](6)$ \\
\hline 618 & 667 & 618 & 24.55 & 111.18 & $\begin{array}{l}{[\omega(29)+\delta(10)](\mathrm{C}=\mathrm{O})+\mathrm{R} 2\left[\delta_{\text {trig }}(11)+\mathrm{oop}(\mathrm{NC} 21)(10)\right]+\rho\left[\mathrm{CH}_{3}\right](6)+\mathrm{R} 1\left[\delta_{\mathrm{a}}\right]} \\
(6)\end{array}$ \\
\hline 592 & 646 & 591 & 26.66 & 239.37 & $\begin{array}{l}{[\omega(13)+\delta(9)](\mathrm{C}=\mathrm{O})+\mathrm{R} 1[\operatorname{puck}(12)+\tau(7)]+\mathrm{R} 2[\mathrm{oop}(\mathrm{NC} 21)(5)+\operatorname{puck}(5)]+} \\
\rho^{\prime}\left[\mathrm{CH}_{3}\right](6)\end{array}$ \\
\hline 581 & 638 & 581 & 45.4 & 58.91 & $\mathrm{R} 2\left[\omega\left(\mathrm{SO}_{2}\right)\right](27)+\mathrm{R} 3\left[\operatorname{puck}(16)+\tau(10)+\delta_{\mathrm{a}}(6)\right]+\mathrm{R} 1\left[\delta^{\prime}{ }_{\mathrm{a}}\right](9)$ \\
\hline 561 & 630 & 561 & 53.14 & 590.6 & $\mathrm{R} 2\left[\delta\left(\mathrm{SO}_{2}\right)(26)+\delta_{\text {trig }}(8)+\delta^{\prime}{ }_{\mathrm{a}}(7)\right]+\mathrm{R} 1\left[\tau^{\prime}\right](5)+\mathrm{R} 3[\tau](5)+\tau(\mathrm{C} 11 \mathrm{C} 12)(5)$ \\
\hline 555 & 606 & 555 & 13.39 & 309.64 & $\begin{array}{l}\mathrm{R} 2\left[\operatorname{puck}(16)+\delta_{\text {trig }}(7)+\rho\left(\mathrm{SO}_{2}\right)(5)\right]+\omega[\mathrm{C}=\mathrm{O}](16)+\mathrm{R} 3[\tau](10)+\mathrm{R} 1[\tau](7)+\rho \\
,\left[\mathrm{CH}_{3}\right](5)\end{array}$ \\
\hline 516 & 567 & 516 & 11.61 & 34.39 & $\mathrm{R} 1[\tau](29)+\mathrm{R} 3[\tau](22)+\mathrm{R} 2[\tau](15)$ \\
\hline 502 & 553 & 502 & 7.58 & 81.73 & $\begin{array}{l}\mathrm{R} 3\left[\tau^{\prime}\right](18)+\delta[\mathrm{C}=\mathrm{O}](14)+\mathrm{R} 2\left[\rho\left(\mathrm{SO}_{2}\right)(12)+\tau^{\prime}(6)\right]+\tau(\mathrm{C} 1 \mathrm{C} 10)(9)+\mathrm{R} 1\left[\tau^{\prime}(7\right. \\
\left.)+\delta_{\mathrm{a}}(6)\right]+\tau(\mathrm{C} 11 \mathrm{C} 12)(5)\end{array}$ \\
\hline 464 & 519 & 464 & 8.81 & 249 & $\begin{array}{l}\mathrm{R} 2\left[\delta\left(\mathrm{SO}_{2}\right)(23)+\mathrm{oop}(\mathrm{NC} 21)(9)+\delta_{\text {trig }}(8)+\tau^{\prime}(7)\right]+\mathrm{R} 3[\tau](16)+\tau(\mathrm{C} 1 \mathrm{C} 10)(1 \\
3)+\delta[\mathrm{C}=\mathrm{O}](5)\end{array}$ \\
\hline 458 & 505 & 458 & 1.63 & 301.3 & $\begin{array}{l}\mathrm{R} 1\left[\tau^{\prime}\right](30)+\mathrm{R} 3\left[\tau^{\prime}(10)+\tau(10)\right]+\tau(\mathrm{C} 11 \mathrm{C} 12)(13)+\tau(\mathrm{C} 1 \mathrm{C} 10)(8)+\mathrm{R} 2[\gamma(\mathrm{SO} \\
2)](6)\end{array}$ \\
\hline 411 & 442 & 412 & 10.79 & 887.25 & $\rho[\mathrm{C}=\mathrm{O}](28)+\mathrm{R} 3\left[\tau^{\prime}(11)+\tau(6)+\delta^{\prime}{ }_{\mathrm{a}}(8)\right]+\mathrm{R} 2[\mathrm{v}(\mathrm{CN})](10)$ \\
\hline 397 & 436 & 398 & 8.74 & 409.44 & $\mathrm{R} 2\left[\delta_{\mathrm{a}}(36)+\delta_{\mathrm{a}}^{\prime}(16)+\mathrm{v}(\mathrm{CS})(10)\right]+\mathrm{R} 1\left[\tau^{\prime}\right](9)$ \\
\hline 381 & 414 & 382 & 2.12 & 727.17 & $\begin{array}{l}\mathrm{R} 1\left[\tau^{\prime}\right](15)+[\rho(14)+\delta(6)](\mathrm{C}=\mathrm{O})+\mathrm{R} 2\left[\rho\left(\mathrm{SO}_{2}\right)(11)+\tau^{\prime}(7)+v(\mathrm{CS})(5)\right]+\mathrm{R} 3[ \\
\tau](5)\end{array}$ \\
\hline 353 & 387 & 354 & 1.29 & 2098.6 & $\mathrm{R} 3\left[\tau^{\prime}(18)+\tau(6)\right]+\mathrm{R} 1\left[\tau^{\prime}\right](15)+\mathrm{R} 2[\mathrm{v}(\mathrm{CS})(6)+\mathrm{v}(\mathrm{CN})(5)]$ \\
\hline 319 & 356 & 320 & 1.12 & 867.18 & $\mathrm{R} 2\left[\delta^{\prime}{ }_{\mathrm{a}}(29)+\delta_{\mathrm{a}}(13)+\mathrm{v}(\mathrm{NC} 21)(5)\right]+[\delta(11)+\rho(5)]\left(\mathrm{SO}_{2}\right)+\mathrm{R} 3\left[\tau^{\prime}\right](9)$ \\
\hline 312 & 347 & 313 & 4.36 & 268.87 & $\begin{array}{l}\mathrm{R} 2\left[(\gamma(15)+\omega(5))\left(\mathrm{SO}_{2}\right)+v(\mathrm{CS})(11)+\delta_{\text {in }}(\mathrm{NC} 21)(8)\right]+\mathrm{R} 1\left[\tau^{\prime}(11)+\tau(9)\right]+\mathrm{R} 3 \\
{\left[\tau^{\prime}\right](6)+\rho[\mathrm{C}=\mathrm{O}](6)}\end{array}$ \\
\hline 280 & 306 & 281 & 1.99 & 197.05 & $\begin{array}{l}\mathrm{R} 3[\tau](19)+\mathrm{R} 1[\tau](11)+\mathrm{R} 2\left[\mathrm{oop}(\mathrm{NC} 21)(6)+\left(\rho(6)+\delta(5)\left(\mathrm{SO}_{2}\right)\right)+v(\mathrm{CS})(7)+\right. \\
\left.\delta_{\text {trig }}(5)\right]+\tau(\mathrm{C} 1 \mathrm{C} 10)(10)+\tau(\mathrm{C} 11 \mathrm{C} 12)(5)\end{array}$ \\
\hline
\end{tabular}


Bhawani Datt Joshi et al. / BIBECHANA 9 (2013) 38-49: BMHSS, p.46 (Online Publication: Nov., 2012)

\begin{tabular}{|c|c|c|c|c|c|}
\hline 242 & 274 & 243 & 4.75 & 1023.84 & $\mathrm{R} 2\left[(\omega(20)+\gamma(18))\left(\mathrm{SO}_{2}\right)+\mathrm{v}(\mathrm{CS})(16)+\delta_{\mathrm{a}}(11)\right]+\mathrm{R} 3\left[\delta_{\mathrm{a}}\right](5)$ \\
\hline 205 & 224 & 206 & 1.75 & 636.7 & $\mathrm{R} 2\left[\gamma\left(\mathrm{SO}_{2}\right)(23)+\delta_{\text {in }}(\mathrm{NC} 21)(18)\right]+\mathrm{R} 3[\tau](15)+\tau(\mathrm{C} 1 \mathrm{C} 10)(14)+\mathrm{R} 1[\tau](6)$ \\
\hline 193 & 214 & 194 & 0.26 & 784.79 & $\begin{array}{l}\mathrm{R} 2\left[\mathrm{oop}(\mathrm{NC} 21)(13)+\rho\left(\mathrm{SO}_{2}\right)(10)+v(\mathrm{CS})(10)+\delta_{\text {trig }}(5)+\tau^{\prime}(5)\right]+\mathrm{R} 1[\tau](12)+ \\
\mathrm{R} 3\left[\delta_{\mathrm{a}}\right](6)\end{array}$ \\
\hline 150 & 168 & 151 & 2.18 & 3124.11 & $\begin{array}{l}\tau(\mathrm{C} 11 \mathrm{C} 12)(28)+\tau(\mathrm{C} 1 \mathrm{C} 10)(25)+\mathrm{R} 2\left[\gamma\left(\mathrm{SO}_{2}\right)(14)+\delta_{\text {in }}(\mathrm{NC} 21)(8)\right]+\mathrm{R} 3\left[\tau^{\prime}\right]( \\
5)\end{array}$ \\
\hline 128 & 147 & 129 & 0.12 & 6552.24 & $\begin{array}{l}\tau(\mathrm{C} 21 \mathrm{C} 22)(26)+\mathrm{R} 2\left[\operatorname{puck}(25)+\rho\left(\mathrm{SO}_{2}\right)(5)\right]+\tau(\mathrm{C} 11 \mathrm{C} 12)(10)+\tau(\mathrm{C} 1 \mathrm{C} 10)( \\
8)+\rho^{\prime}\left[\mathrm{CH}_{3}\right](6)+\mathrm{R} 3[\tau](6)\end{array}$ \\
\hline 115 & 131 & 116 & 0.2 & 5116.14 & $\tau(\mathrm{C} 21 \mathrm{C} 22)(29)+\tau(\mathrm{C} 11 \mathrm{C} 12)(22)+\mathrm{R} 2\left[\operatorname{puck}(11)+\mathrm{R} 2 \tau^{\prime}(7)\right]+\rho^{\prime}\left[\mathrm{CH}_{3}\right](8)$ \\
\hline 97 & 114 & 98 & 0.94 & 1060.01 & $\begin{array}{l}\mathrm{R} 2[\tau](26)+\mathrm{R} 2\left[\tau^{\prime}(12)+\delta_{\text {in }}(\mathrm{NC} 21)(7)\right]+\tau(\mathrm{C} 11 \mathrm{C} 12)(18)+\tau(\mathrm{C} 21 \mathrm{C} 22 \\
)(10)+\tau(\mathrm{C} 1 \mathrm{C} 10)(7)\end{array}$ \\
\hline 75 & 81 & 76 & 0.59 & 19405.86 & $\begin{array}{l}\mathrm{R} 2\left[\mathrm{oop}(\mathrm{NC} 21)(45)+\tau^{\prime}(5)\right]+\tau(\mathrm{C} 1 \mathrm{C} 10)(16)+\tau(\mathrm{C} 21 \mathrm{C} 22)(10)+\tau(\mathrm{C} 11 \mathrm{C} 12) \\
(6)\end{array}$ \\
\hline 71 & 76 & 71 & 4.07 & 1737.43 & $\tau(\mathrm{C} 21 \mathrm{C} 26)(63)+\mathrm{R} 2\left[\delta_{\text {in }}(\mathrm{NC} 21)\right](5)+\tau(\mathrm{C} 1 \mathrm{C} 10)(5)$ \\
\hline 51 & 50 & 52 & 4.64 & 23135.09 & $\left.\mathrm{R} 2\left[\tau^{\prime}\right](24)+\mathrm{oop}(\mathrm{NC} 21)(14)+\tau(5)\right]+\tau(\mathrm{C} 21 \mathrm{C} 26)(18)+\tau(\mathrm{C} 21 \mathrm{C} 22)(14)$ \\
\hline
\end{tabular}

\subsection{2. $\mathrm{C}=\mathrm{O}$ vibrations}

A carbonyl $(\mathrm{C}=\mathrm{O})$ group is connected at the nitrogen atom of ring $\mathrm{R} 2$. Regarding to this group; stretching, bending, rocking and wagging modes of vibrations are observed. The stretching of $\mathrm{C}=\mathrm{O}$ is calculated at $1725 \mathrm{~cm}^{-1}$ (contribution 78\%). The deformation and wagging calculated at 618 and $591 \mathrm{~cm}^{-1}$ are the mixed modes. The rocking vibration is calculated at $412 \mathrm{~cm}^{-1}$ (contribution $28 \%$ ) with the intensities of 10.79 a.u. in the IR spectrum and 887.25 a.u. in the Raman spectrum, respectively.

\subsubsection{Ring R1 vibrations}

Ring R1 has four $\mathrm{CH}$ moieties; hence one can expect four stretching modes associated to this group. All these modes are pure in the range 3090-3040 $\mathrm{cm}^{-1}$ and have very weak in intensity in the IR spectrum. The mixed in-plane deformations are calculated at 183, 1468, 1454, 1293, 1174 and $1147 \mathrm{~cm}^{-1}$. The outof-plane bending modes are calculated at 994, 963, 878 and $769 \mathrm{~cm}^{-1}$.

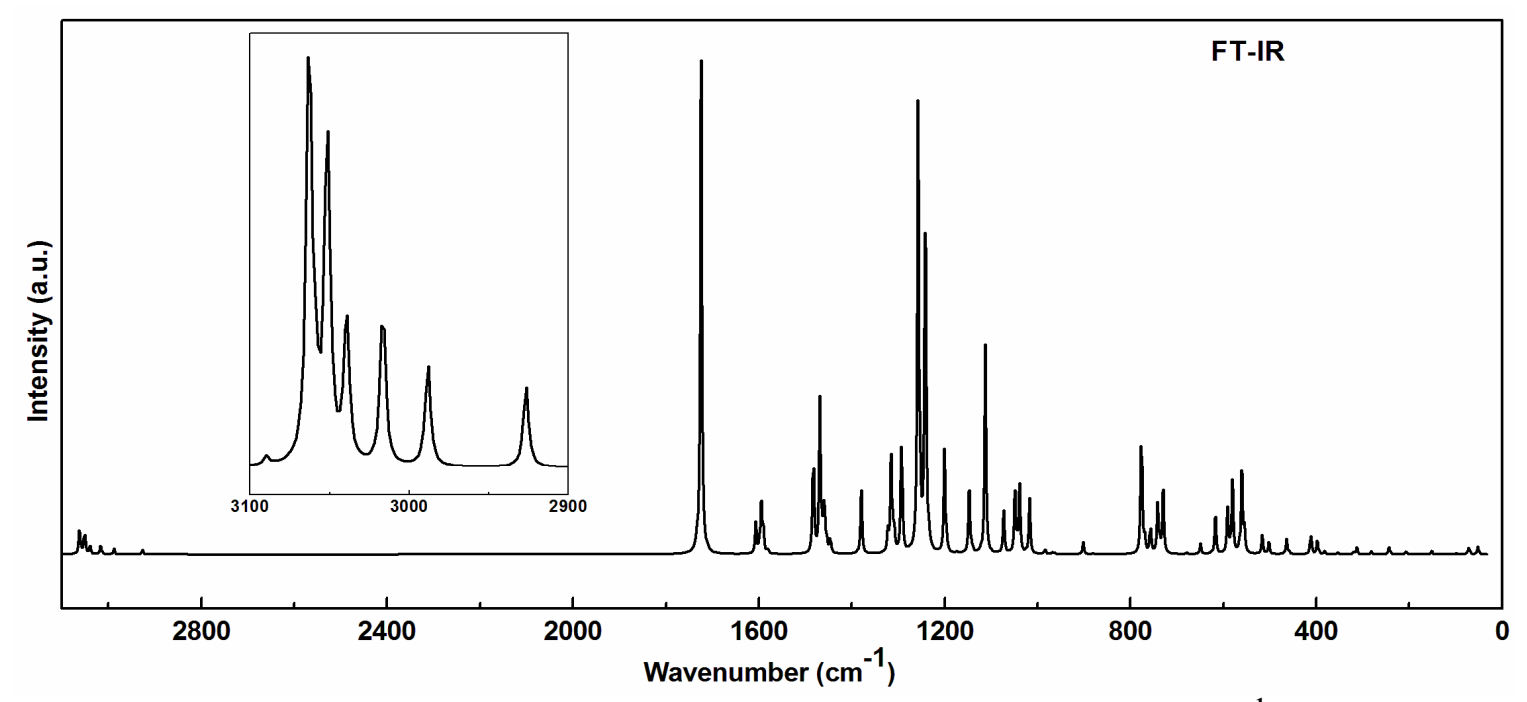

Fig. 5. Theoretical IR spectra between the ranges $0-3100 \mathrm{~cm}^{-1}$. 
The C-C stretching is stronger in intensity in the Raman spectrum than in the IR spectrum.

These modes are calculated below $1595 \mathrm{~cm}^{-1}$. Around $741 / 730 \mathrm{~cm}^{-1}$ and $737 / 678 \mathrm{~cm}^{-1}$ are the puckering and deformation of the ring, respectively. The ring torsion is calculated at 516,458 and $382 \mathrm{~cm}^{-1}$ in the mixed modes.

\subsubsection{Ring $R 2$ vibrations}

Two important functional groups $\mathrm{SO}_{2}$ and $-\mathrm{COCH}_{3}$ are connected with this ring $\mathrm{R} 2$. The six fundamental modes of vibrations are assigned with $\mathrm{SO}_{2}$ group; namely symmetric, asymmetric stretch, deformation and rocking, which belong to polarized in-plane vibrations. In addition to that, $\mathrm{SO}_{2}$ wagging and twisting modes would be expected to be depolarized out-of-plane symmetry species. The $\mathrm{SO}_{2}$ asymmetric stretching having contribution of $85 \%$ in PED is calculated at $1257 \mathrm{~cm}^{-1}$. The symmetric stretching is calculated at $1113 / 1029 \mathrm{~cm}^{-1}$, which have strong Raman intensities of $1162.02 / 1154.47$ units. The deformations are calculated at 561 and $464 \mathrm{~cm}^{-1}$. The wagging and twisting vibrations are calculated at $581 / 243 \mathrm{~cm}^{-1}$ and $313 / 206 \mathrm{~cm}^{-1}$, respectively. Highly mixed rocking mode with low contribution in PED is calculated below $555 \mathrm{~cm}^{-1}$.

$\mathrm{CN}$ stretching is calculated at 1315 and $1200 \mathrm{~cm}^{-1}$. NC21 stretching is calculated at $1243 \mathrm{~cm}^{-1}$, which has weak IR and strong Raman intensities as listed in Table 2.

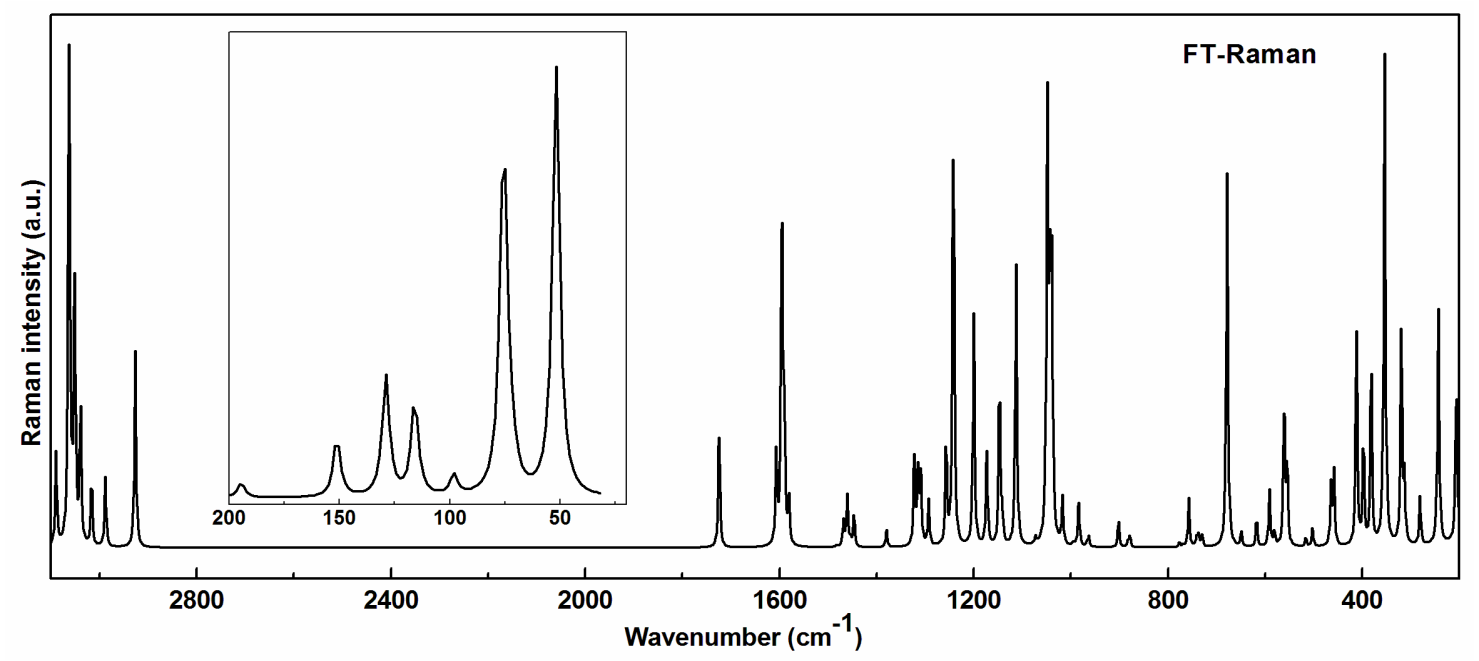

Fig. 6. Theoretical Raman spectra between the ranges 20-3200 cm-1.

\subsubsection{Ring $R 3$ vibrations}

Ring R3 has also four $\mathrm{CH}$ moieties; hence four $\mathrm{CH}$ stretching modes are assigned between the range $3063-3039 \mathrm{~cm}^{-1}$. The CC stretching of the ring is calculated at $1607 / 1048 \mathrm{~cm}^{-1}$. The trigonal ring deformation is calculated at $1073 \mathrm{~cm}^{-1}$ with weak intensities both in the IR and the Raman spectra. The ring puckering, asymmetric deformation and the ring torsion are calculated at 757, 649 and $502 \mathrm{~cm}^{-1}$, respectively.

\section{Conclusion}

The equilibrium geometries and harmonic vibrational wavenumbers of all the 84 normal modes of the APTZ molecule were determined and analyzed both at DFT (B3LYP) and HF levels of theory employing the $6-311++\mathrm{G}(\mathrm{d}, \mathrm{p})$ basis set. These theoretical vibrational assignments along with the electronic transitions are important to understand the molecular structure and biological activity of the title 
molecule. The IR and the Raman spectra were presented, and the vibrational bands were assigned on the basis of the PED obtained from the DFT calculations. Information about the size, shape, charge density distribution and structure-activity relationship of the APTZ molecule has been obtained by mapping electron density isosurface with ESP and MESP. HOMO - LUMO made very clearly the involvement of charge transfer between the donor and acceptor groups.

\section{Acknowledgements}

The authors are grateful to the University Grants Commission, Nepal, and Alexander von Humboldt Foundation, Germany, for providing partial financial assistance.

\section{References}

[1] S.A. Alkalis, G. Beck and M. Grätzel, J. Am. Chem. Soc., 97 (1975) 5723.

[2] Y. Dixit, R. Dixit, N. Gautam and D.C. Gautam, E-J. Chem., 5(S1) (2008) 1063.

[3] C. Chan, H. Yin, J. Garforth, J.H. McKie, R. Jaouhari, P. Speers, K.T. Douglas, P.J. Rock, V. Yardley, S.L. Croft, and A.H. Fairlamb, J. Med. Chem., 41 (1998) 148.

[4] B.R. Henry and M. Kasha, J. Chem. Phys., 47 (1967) 3319.

[5] M.A. Wuonola, M.G. Palfreyman, N. Motohashi, M. Kawase, S. Gabay, R.R. Gupta and J. Molnar, Anticancer Res., 18 (1998) 337.

[6] J. Molnar, A. Hever, I. Fakla, J. Fischer, I. Ocsovaki, and A. Aszalos, Anticancer Res., 17 (1997) 481.

[7] M.A. Wuonola, M.G. Palfreyman, N. Motohashi, M. Kawase, S. Gabay and J. Molnar, J. Anticancer Res., 17 (1997) 3425.

[8] M.A. Wuonola, M.G. Palfreyman, N. Motohashi, M. Kawase, S. Gabay, J. Nacsa, and J. Molnar, J. Anticancer Res., 17 (1997) 3409.

[9] I.K. Pajeva and M. Wiese, Quant. Struct-Act. Relat. ,16 (1997) 1.

[10] I.K. Pajeva, M. Wiese, H.P. Cordes, and J.K. Seydel, J. Cancer Res. Clin. Oncol., 122 (1996) 27.

[11] R.A. Singh, R. Singh, O.S. Rao, and S.M. Verma, Mol. Cryst. Liq. Cryst., 237 (1993) 419.

[12] M. Pampallona, A. Ricci, B. Scrosati and C.A. Vincent, J Appl Electrochem., 6 (1976) 269.

[13] R. Singh and R.A. Singh, Mol. Cryst. Liq. Crys.t Sci. Technol. Sect., 8C (1997) 187.

[14] M. Alconea Palafox, M. Gil, J.L. Núñez and G. Tardajos, Int. J. Quant. Chem., 89 (2002) 147.

[15] R. Sharma, P. Samadhiya, S.D. Srivastava and S.K. Srivastava, J. Serb. Chem. Soc., 77(1) (2012) 17.

[16] B.D. Joshi, A. Srivastava, P. Tandon and S. Jain, Spectrochim. Acta. A, 82 (2011) 270.

[17] Q. Wang, L. Yang, Z. Xu and Y. Sun, Acta Cryst. E,65 (2009) 1978.

[18] P. Hohenberg and W. Kohn, Phys. Rev. B, 136B (1964) 864.

[19] M.J. Frisch, G.W. Trucks, H.B. Schlegel. G.E. Scuseria, J.R. Cheeseman, M.A. Robb, G. Scalmani, V. Barone, B. Mennucci, G.A. Petersson, H. Nakatsuji, M. Caricato, X. Li, H.P. Hratchian, A.F. Izmzylov, J. Bloino, G. Zheng, J.L. Sonnenberg, M. Hada, M. Ehara, K. Toyota, R. Fukuda, J. Ishida, M. Hasegawa, T. Nakajima, Y. Honda, O. Kitao, H. Nakai, T. Vreven, J.A. Montgomery, Jr., J.E. Peralta, F. Ogliaro, M. Bearpark, J.J. Heyd, E. Brothers, K.N. Kudin, V.N. Staroverov, R. Kobayashi, J. Normand, A. Raghavachari, A. Rendell, J.C. Burant, S.S. Iyengar, J. Tomasi, M. Cossi, N. Rega, J.M. M illan, M. Klene, J.E. Knox, J.B. Cross, V. Bakken, C. Adamo, J. Jaramillo, R. Gomperts, R.E. Stratmann, O. Yazyev, A.J. Austin, R. Cammi, C. Pomelli, J.W. Ochterski, R.L. Martin, K. Morokuma, V.G. Zakrzewski, G.A. Voth, P. Salvador, J.J. Dannerberg, S. Dapprich, A.D. Daniels, J. Farkas, B. Foresman, J.V. Ortiz, J. Cioslowski, and D.J. Fox, GAUSSIAN 09, Revision, Gaussian, Inc., Wallingford CT, 2009. C.T. Lee, W.T. Yang and R.G. Parr, Phys. Rev. B, 37 (1988) 785.

A.D. Becke, J. Chem. Phys., 98 (1993) 5648.

[22] G.A. Petersson and M.A. Allaham, J. Chem. Phys., 94 (1991) 6081.

[23] G.A. Petersson, A. Bennett, T.G. Tensfeldt, M.A. Allaham and W.A. Shirley, J. Mantzaris, J. Chem. Phys., 89 (1988) 2193.

[24] P. Pulay, G. Fogarasi, F. Pang and J.E. Boggs, J. Am. Chem. Soc., 101 (1979) 2550.

[25] G. Fogarasi, X. Zhou, P.W. Taylor and P. Pulay, J. Am. Chem. Soc., 114 (1992) 8191. 
Bhawani Datt Joshi et al./ BIBECHANA 9 (2013) 38-49: BMHSS, p.49 (Online Publication: Nov., 2012)

[26] J.M.L. Martin and C.V. Alsenoy, Gar2ped, University of Antwerp (1995).

[27] G.A. Zhurko and D.A. Zhurko, Chemcraft (2005).

[28] P. Politzer and D. G. Truhlar, Chemical Applications of Atomic and Molecular Electrostatic Potentials, Plenum, New York (1981).

[29] S. R. Gadre, P. K. Bhadane, S. S. Pundlik and S. S. Pingale, Molecular Electrostatic Potentials Concepts and Applications, ed. J. S. Murray and K. D. Sen (1996) pp. 219.

[30] P. Politzer, M. E. Grice, J. S. Murray and J. M. Seminario, Can. J. Chem., 71 (1993) 1123.

[31] C. H. Suresh, P. Alexander, K. P. Vijayalakshmi, P. K. Sajith and S. R. Gadre, Phys. Chem. Chem. Phys., $10(2008) 6492$.

[32] R.M. Issa, M.K. Awad and F.M. Atlam, Appl. Surf. Sci., 255 (2008) 2433.

[33] H. Yoshida, K. Takeda, J. Okamura, A. Ehara, and H. Matsurra, J. Phys. Chem. A, 106 (2002) 3580.

[34] M.W. Wong, Chem. Phys. Lett., 256 (1996) 391.

[35] A.P. Scott and L. Radom, J. Phys. Chem., 100 (1996) 16502.

[36] G.A. Guirgis, P. Klaboe, S. Shen, D.L. Powell, A. Gruodis, V. Aleksa, C.J. Nielsen, J. Tao, C. Zheng and J.R. Durig, J. Raman Spectrosc., 34 (2003) 322.

[37] P.L. Polavarapu, J. Phys. Chem., 94 (1990) 8106. 